\title{
Is Duplex-Ultrasound a useful tool in defining rejection episodes in composite tissue allograft transplants?
}

\author{
Alexander Loizides ${ }^{1}$, Irmgard-Elisabeth Kronberger², Michaela Plaikner¹, Hannes Gruber ${ }^{1}$
}

${ }^{1}$ Department of Radiology, ${ }^{2}$ Department of Visceral, Transplant and Thoracic Surgery, Innsbruck Medical University, Innsbruck, Austria

\begin{abstract}
Immunologic reactions in transplanted organs are in more or less all allograft patients detectable: clear parameters exist as e.g. in renal transplants where the clearance power reduces by rejection. On the contrary, in composite tissue allografts clear and objective indicators stating a rejection episode lack. We present the case of a hand-transplanted subject with signs of acute transplant rejection diagnosed by means of Duplex Ultrasound and confirmed by biopsy.
\end{abstract}

Keywords: Duplex-Ultrasound, hand-transplant, composite tissue allografts, rejection

\section{Introduction}

As in all allograft patients there are more or less marked episodes of "immunologic stress" in transplanted organs. According immunologic algorithms and their interaction to whatever immun-modulating medication are initiated: as e.g. in renal transplants the clearance power reduces by rejection [1-3]. In contrary, in complex composite graft as a hand - where completely different tissues interact - clear and objective indicators stating a rejection episode lack. Vice versa, after having adapted an immunemodulating therapy, there is no clear indicator for controlling success or failure of such a measure. To date an easy tool is lacking to do this, as biopsy is the Golden standard for assessing the above mentioned: however, biopsy is a slow, an immanent risky (infection) and an often not that easily to interpret tool before all in the assessment of correct measures against rejection taken. It is known, that the first areas of "immunologic change“

Received 08.06.2015 Accepted 20.06.2015

Med Ultrason

2015, Vol. 17, No 4, 550-552

Corresponding author: Alexander Loizides, MD

Innsbruck Medical University,

Department of Radiology

Anichstrasse 35, 6020 Innsbruck, Austria

Phone: $+43 / 512 / 504 / 22761$

Fax: $+43 / 512 / 504 / 22758$

E-mail: alexander.loizides@i-med.ac.at in any transplant are vascular structures [4,5]: immune complexes can be found in the (sub)intimal space where initial interaction between the patient's immune system and foreign tissue/material occur; these - if mild and stable - do actually not hamper the graft severely and may vanish by time. However, in cases of (acute) rejection episodes, these (sub)intimal plaques and thickenings get "out of control" and may even reach a size, that markedly impairs the (arterial) blood flow primarily and secondarily by promoting clotting of the blood. Accordingly, changes in whatever tissue of the graft start and proceed with the potential of irreversibly destroying functionally necessary parts of the graft [6].

In the last decade, high-resolution ultrasonography (HRUS) and especially Duplex ultrasound (DU) have become an important adjunct in the evaluation of vascular pathologies [7]. With high-frequency broadband transducers and highly sensitive color and power Doppler the blood flow and the structure of vessels measuring at least $100 \mu \mathrm{m}$ in diameter can be characterized [8]. Therefore, this modality was expected to also have a potential for diagnosing vascular impairment in patients where a rejection (with its vascular changes) is suspected.

We present the case of a hand-transplanted subject, who presented with signs of acute transplant rejection: we had the opportunity of doing a HRUS including DU and Doppler-spectrum wave analysis at the time periodically before and at the time of rejection (including the photo- 
documentation of the cutaneous changes); our findings (as a singular observation) might at least serve as a rather powerful adjunct in handling such complex patients.

\section{Case report}

A 60 year old male patient who underwent a unilateral hand composite tissue allograft (CTA) transplantation at the right side 6 years ago presented with diffuse swelling of the allograft (clinical lymphatic edema) with progressive impairment of finger motion (extension and flexion). Preliminary data on perfusion of the ulnar and radial artery was available due to periodical controls of the subject including thorough HRUS and DU assessment. Additionally the subject presented a diffuse scarlitiform exanthema of the transplant with a proximo-distal gradient (fig 1a). The patient was referred to our department for a HRUS evaluation of the graft under suspicion of rejection; additionally the patient underwent a biopsy at the time of suspected rejection.

\section{Preliminary sonographic assessment:}

- Grey scale sonography showed some mild subcutaneous and intermuscular clefts with anechoic fluid collections. Some minor (hyper)echoic texture changes of the lower-arm muscles and some slight effusions of the tendon sheaths were seen.

- DU showed preserved vascularity of the muscles; the ulnar and the radial artery showed orthograde perfusion without marked thickened walls or focal intima hyperplasia.

- The Doppler spectra (built always at the same position at the radiocarpal joint) presented rather low resistance indices always $<0.8$ with rather lengthened acceleration times $>100 \mathrm{~ms}$ : as this was consistent with other allografts, this situation was accepted as the "normal post-transplant" state (fig 1b).

\section{Sonographic assessment under suspicion of rejection:}

- Grey scale sonography showed widened subcutaneous and intermuscular clefts and spaces with disperse anechoic fluid collections. Additionally, slight hyperechoic texture changes of the muscles were obvious including slight effusions of the tendon sheaths.

- DU showed preserved vascularity of the muscles; the ulnar and the radial artery showed orthograde perfusion without marked thickened walls or spotty humps as inflammatory intima hyperplasia were obvious.

- The Doppler spectra built by again setting the „detection volume" (DV; spectrum relevant gate) at the radiocarpal and ulnocarpal segment of the radial and ulnar artery, respectively, presented relatively reduced acceleration times and markedly elevated resistance indices $=$ 1 (fig 1c).

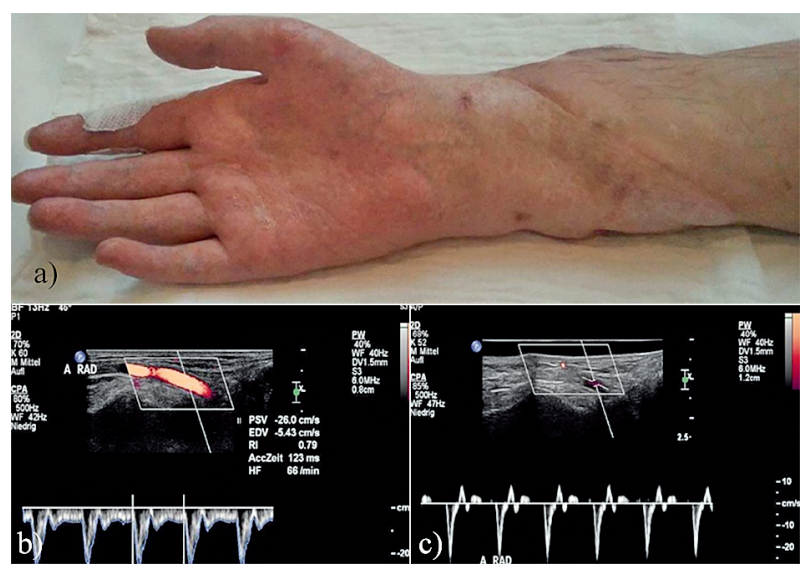

Fig 1. a) Diffuse scarlitiform exanthema of the transplant with a proximo-distal gradient of a 60 year old male patient who underwent a unilateral hand composite tissue allograft (CTA) transplantation at the right side; b) preliminary sonogram with a "normal post-transplant" Doppler spectrum presenting a low resistance index $<0,8$ and a rather lengthened acceleration time $>100 \mathrm{~ms}$; c) sonogram under suspicion of rejection with a Doppler spectrum presenting a markedly elevated resistance index $=1$ and a relatively reduced acceleration time.

\section{Histology Assessment of biopsy of the graft: Mild rejection reaction BANFF 0-1. \\ Secondary Evaluation:}

After treatment with $250 \mathrm{mg}$ Urbason on three consecutive days and after increasing the basic-immune-suppression on a Tacrolimus-level of 4-6 $\mathrm{ng} / \mathrm{dl}$ for one week the patient presented an amelioration of the impaired finger motion and a visible improvement of the scarlitiform exanthema. In the next standard follow-up HRUS examination the Doppler spectra were "normalized" to the "normal post-transplant" state.

\section{Discussions}

Composite tissue allograft is a rather rare but recent topic in transplant surgery. Reliable parameters indicating acute graft rejection are still ill defined. To date acute rejection episodes of the graft are characterized clinically, by blood test, or by biopsy: patients present cutaneous lesions of the graft with or without edema $[9,10]$.

As one of the first immunologic changes in an acute graft rejection is on a vascular level, an additional or rather alternative method besides the invasive biopsy is needed for diagnosing the vascular changes presented in a rejection episode. In this context HRUS could play an important role: HRUS can - due to the adjunct of Duplex-imaging basing on the "Doppler-physics“ - easily detect and depict blood flow down to relatively small arterial and venous blood vessels: it can not only show 
flow in a vessel and by color-coding its direction, but can also clearly define the spectrum of the flow at a discrete segment of a vessel. The spectrum gives several clear information not only on the blood flow at a point of measurement (POM) but also on the flow proximal and distal to it: stenosis proximal to a POM appears as an elongation of blood flow acceleration time, stenosis at a POM appears as a higher peak velocity and stenosis distal to a POM reflects as a higher resistance index. Ostermann et al were one of the first researchers who described the usefulness of HRUS in the evaluation of patients who had undergone a CSA transplantation: they evaluated by means of HRUS vascular changes e.g. resistance indices, wall thickening, wall insufficiency (aneurysm), arterial vascular occlusion and venous changes [11] and could show in the rather limited number of cases, that atypically low resistance indices $(\mathrm{RI}<1)$ are evident in clinically "healthy" grafts. Basing on these data the mildly elevated $\mathrm{AT}$ and markedly elevated resistance indices $(\mathrm{RI}=1)$ of a clinically "pathological" graft led to the suspected acute rejection diagnose which was confirmed by biopsy.

In summary Duplex ultrasound can help in stating a "pathological" finding in this admittedly very special field and should therefore be the first-line-modality in patients with CSA transplantation and suspected acute rejection.

\section{References}

1. Roberts DM, Jiang SH, Chadban SJ. The treatment of acute antibody-mediated rejection in kidney transplant recipientsa systematic review. Transplantation 2012; 94: 775-783.

2. Gupta G, Abu Jawdeh BG, Racusen LC, et al. Late antibody-mediated rejection in renal allografts: outcome after conventional and novel therapies. Transplantation 2014; 97: 1240-1246.

3. Marcen R, Dal Canton A. Glomerular filtration rate: utility for assessing long-term renal allograft outcomes in kidney allograft recipients. J Nephrol 2013; 26: 1009-1024.

4. Rowaiye OO, Kusztal M, Klinger M. The kidneys and ANCA-associated vasculitis: from pathogenesis to diagnosis. Clin Kidney J 2015; 8: 343-350.

5. Mor E, Lustig S, Tovar A, et al. Thrombotic microangiopathy early after kidney transplantation: hemolytic uremic syndrome or vascular rejection? Transplant Proc 2000; 32: 686-687.

6. Filippone EJ, Farber JL. The specificity of acute and chronic microvascular alterations in renal allografts. Clin Transplant 2013; 27: 790-798.

7. Bodner G, Schocke MF, Rachbauer F, et al. Differentiation of malignant and benign musculoskeletal tumors: combined color and power Doppler US and spectral wave analysis. Radiology 2002; 223: 410-416.

8. Therasse P, Arbuck SG, Eisenhauer EA, et al. New guidelines to evaluate the response to treatment in solid tumors. European Organization for Research and Treatment of Cancer, National Cancer Institute of the United States, National Cancer Institute of Canada. J Natl Cancer Inst 2000; 92: 205-216.

9. Lanzetta M, Petruzzo P, Dubernard JM, et al. Second report (1998-2006) of the International Registry of Hand and Composite Tissue Transplantation. Transpl Immunol 2007; 18: 1-6.

10. Petit F, Minns AB, Dubernard JM, Hettiaratchy S, Lee WP. Composite tissue allotransplantation and reconstructive surgery: first clinical applications. Ann Surg 2003; 237: 1925.

11. Ostermann S, Loizides A, Spiss V, Peer S, Gruber H. Notable features in composite tissue allografts: value of highresolution ultrasonography as a first-line imaging modality. Ultraschall Med 2011; 32 Suppl 2: E1-E7. 